\title{
Breast Metastases from Ovarian Primary: A Rare Entity and Review of Literature
}

\section{Suresh Singh ${ }^{1}$, Bhalgat Bhushan Sanjay ${ }^{2 *}$, Pravin Kumar ${ }^{2}$, Kamal Kishore Lakhera ${ }^{3}$, Phanindra Kumar Swain $^{2}$ and Raj Govind Sharma ${ }^{4}$}

\author{
${ }^{1}$ Head of Department, Department of Surgical Oncology, SMS Medical College and Hospital, Jaipur, India \\ ${ }^{2}$ Senior Resident, Department of Surgical Oncology, SMS Medical College and Hospital, Jaipur, India \\ ${ }^{3}$ Assistant Professor, Department of Surgical Oncology, SMS Medical College and Hospital, Jaipur, India \\ ${ }^{4}$ Senior Professor, Department of Surgical Oncology, SMS Medical College and Hospital, Jaipur, India
}

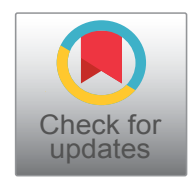

*Corresponding author: Bhalgat Bhushan Sanjay, Senior Resident, Department of Surgical Oncology, SMS Medical College and Hospital, Jaipur, India, Tel: +917083144710

\section{Introduction}

Ovarian cancer is the fifth most frequent diagnosis of female malignancy and the second most common female gynecologic malignancy after breast cancer $[1,2]$. The 5 -year prevalence is 213 cases per million with 2.1 percent deaths as per GLOBOCON 2020 data [3]. Clinical manifestations commonly include abdominal and pelvic pain with bloating, distention, urinary urgency and frequency, and unintended weight loss in the presence of tenderness to palpation and an adnexal mass on bimanual examination. Risk factors for the development of ovarian cancer include early menarche, late menopause, nulliparity, and infertility. Additional risk factors include the BRCA1 and BRCA2 (breast cancer 1 and 2 gene) mutations, as well as Lynch syndrome. Ovarian epithelial cancers spread primarily by exfoliation of cells through the peritoneal cavity, and a minority of cases spread by lymphatic dissemination or haematogenous spread. The most common sites of metastasis are the bowel, peritoneum, and omentum [4]. Metastasis to the breast from extramammary malignancies is rare; representing $0.2-1.3 \%$ of all malignant tumors diagnosed in the breast, [1] and arises most frequently from melanomas, sarcomas, lung cancer, ovarian tumors, and renal carcinomas [5]. Unfortunately imaging findings are not specific and diagnostic to differentiate primary or secondary etiology and no features are characteristic of different metastasis [5]. Histologically, the basis to the diagnosis of metastases of ovarian cancer to breast is that the papillary architecture consistent with serous papillary carcinoma is not a typical pattern of the majority of histological types of invasive breast carcinoma [6]. In addition to histology, immunohistochemistry is frequently used to discern metastases of ovarian cancer to breast from other extramammary breast metastases and primary breast cancers. For example, the expression of WT-1, paired box 8 (PAX8) and mesothelin has been described as being useful in this regard [6] (Figure 1).

\section{Case Report}

A 45-year-old female came with complains of lump in lower abdomen since 3 months. It was insidious in onset and increased gradually over 3 months. It was not associated with any history of pain in abdomen or per vaginal discharge. Her age at menarche was 14 years. Her menstrual cycle frequency was an average of 30 days and lasted for 5 days with average amount of bleeding soaking two pads per cycle. There was no history of intermenstrual or post-coital bleeding. On examination, there was a solitary lump in lower abdomen, which was well defined, firm in consistency and mobile on $\mathrm{P} / \mathrm{V}$ examination. Patient was investigated and found to have bilateral carcinoma ovary with pleural effusion on radiological examination. She underwent

Citation: Singh S, Sanjay BB, Kumar P, Lakhera KK, Swain PK, et al. (2021) Breast Metastases from Ovarian Primary: A Rare Entity and Review of Literature. Int J Oncol Res 4:030. doi.org/10.23937/26434563/1710030

Accepted: July 22, 2021: Published: July 24, 2021

Copyright: (C) 2021 Singh S, et al. This is an open-access article distributed under the terms of the Creative Commons Attribution License, which permits unrestricted use, distribution, and reproduction in any medium, provided the original author and source are credited. 


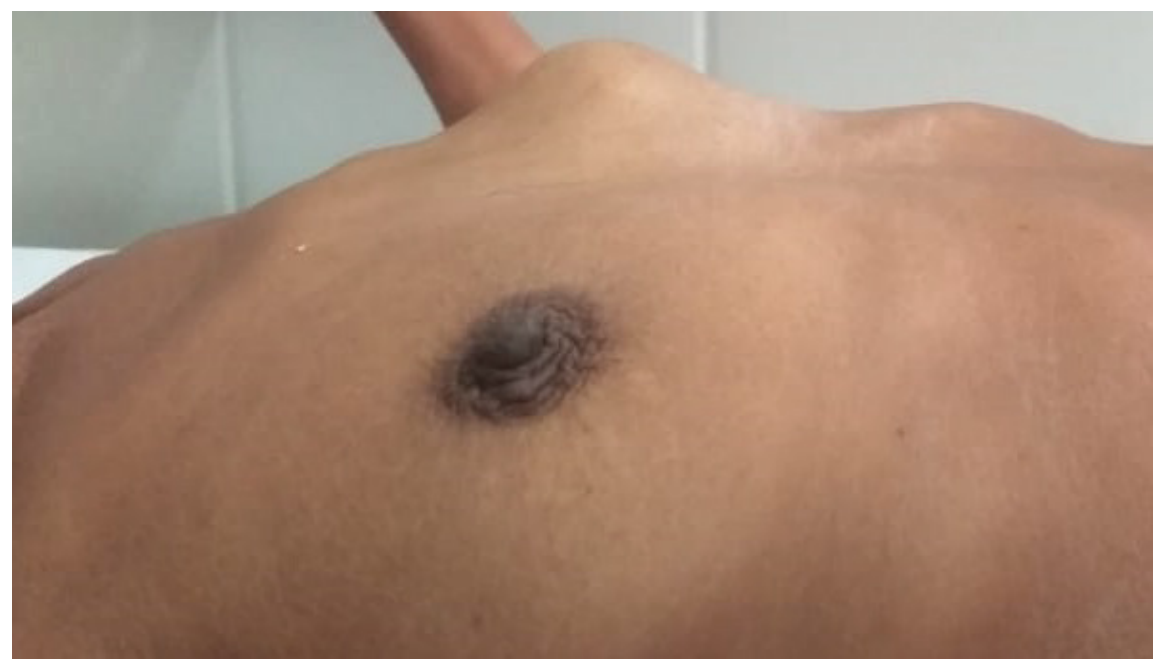

Figure 1: Breast metastases from ovarian carcinoma as seen from the right lateral side.

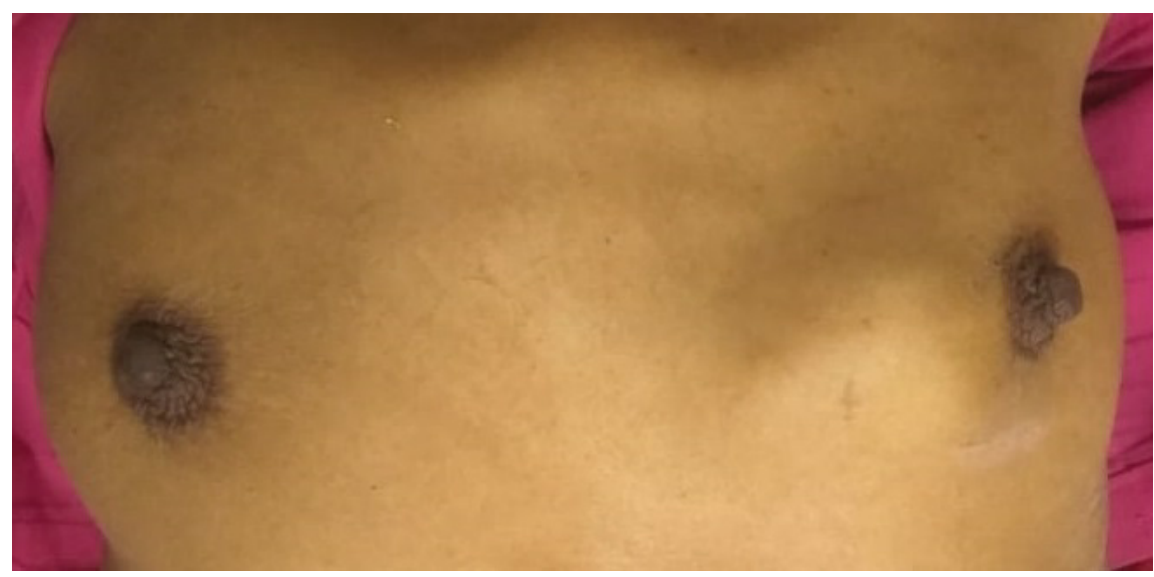

Figure 2: Breast metastases from ovarian carcinoma as seen from the anterior side.

diagnostic laparoscopy with omental biopsy since the disease was found to be advanced. Later after taking 3 chemotherapy cycles of cisplatin and gemcitabine, repeat radiological investigations revealed regressed disease and she was operated for total abdominal hysterectomy with bilateral salpingo-oophorectomy with omentectomy and peritonectomy. Histopathology revealed serous cystadenocarcinoma of both ovaries with omental deposits with positive pelvic lymph nodes on both sides. After taking 3 cycles of post-operative chemotherapy, she presented with complain of lump in left breast. Biopsy of the lump revealed moderately differentiated adenocarcinoma with positivity for CA 125 and cytokeratin (Figure 1 and Figure 2).

\section{Discussion}

Breast cancer is one of the most common primary malignancies in women, yet metastatic tumors to the breast are infrequent, accounting for only $0.5 \%$ to $1.3 \%$ of breast cancer cases [7]. The most common source of metastasis to the breast is a contralateral primary breast tumor, frequently from transthoracic or lymphatic spread. A study by Hadju and Urban involving 4,051 breast cancer patients found an overall incidence of primary gynecologic cancers metastatic to the breast of $0.17 \%$, with only $0.07 \%$ of metastatic disease originating from a primary ovarian tumor [8]. The first case report of ovarian cancer with metastasis to the breast was in 1907 by Sitzenfrey [7]. Serous papillary carcinoma is the most common type of ovarian tumor that can metastasize to the breast [5] and is usually detected within 2 years from the initial diagnosis of primary ovarian cancer. The most common location of metastases is the upper outer quadrant in $62 \%$ of patients [8].

In a study, DeLair, et al. analyzed 85 cases of non-mammary metastases to the breast and axilla, 14 of which were metastases of ovarian cancer to breast [9]. Notably, the ovaries were the predominant site of origin among all carcinomas causing metastases to the breast, comprising $58 \%$ of all cases. Another study by Karam, et al. investigated 29 ovarian cancer patients with malignant breast lesions, 10 of whom exhibited metastases of ovarian cancer to breast and 19 of whom presented with primary breast cancer [10]. The study found marked differences between the two groups regarding disease-free survival time and the mean time interval between the diagnosis of ovarian cancer and the diagnosis of the breast tumor. 
The differential diagnosis between primary breast cancer and a metastatic breast tumor from ovarian cancer is not simple [11]. An accurate clinical history is crucial considering the simultaneous or previous diagnosis of extramammary malignancy, combined with a careful clinical examination, and radiological evaluation, to ensure the correct diagnosis and the most appropriate management of these patients.

The mode of dissemination might originally be drainage from the intra-abdominal to axillary lymph nodes, and then the lymphatic channels of the breast. Generally, hematogenous disseminated lesions are circumscribed masses and may mimic benign masses or circumscribed malignant tumor. Instead, lymphatic dissemination may lead to diffuse breast involvement, edema, trabecular thickening, and skin thickening which may mimic inflammatory process such as mastitis or inflammatory carcinoma [5].

Radiographically, mammographic evaluation can provide additional information. An important histological difference between ovarian and breast cancer is the type of calcifications. Ovarian has psammomatous calcifications whereas breast has non-psammomatous calcifications [5]. Metastatic tumors to the breast more frequently present as well-circumscribed, non-calcified dense masses. They generally lack spiculation and microcalcifications as well as architectural distortion and other skin changes. However, because of the presence of psammoma bodies associated with some ovarian cancers, microcalcifications can be seen with ovarian metastasis. Breast metastasis from a primary ovarian tumor, however, commonly lacks a characteristic pattern, may be morphologically indistinguishable from its primary, and is associated with widespread dissemination [5].

Both primary and metastatic breast cancer can yield similar pathologic pictures and must be distinguished, because primary treatment and survival are significantly different. Immunohistochemistry may provide additional insight into the origin of the tumor. The recognition and distinction between primary and metastases require careful consideration. WT1, PAX8, and GCDFP-15 play a major role in distinguishing between primary and metastatic breast cancers from ovarian cancer, and over $90 \%$ of ovarian serous carcinomas are WT1 positive. Less than $10 \%$ of primary breast cancer cases are positive for WT1, with weak and patchy WT1 expression. However, nuclear reactivity is observed in approximately $20 \%$ of clear cell carcinoma [11]. Hence, negative WT1 in this breast specimen could not distinguish primary versus metastatic breast cancer. PAX8 is positive in most ovarian nonmucinous surface epithelial tumors: Serous, clear cell, and endometrioid cell types, especially for endometrioid and clear cell carcinoma, in which WT1 is generally negative or only focally positive. In a recent immunohistochemistry study, GATA3 showed superior sensitivity compared to GCDFP-15 in detecting primary breast origin. GCDFP-15 and GATA3 could be used to make a distinction between primary breast and metastatic ovarian cancer [9].

Mammary and non-mucinous ovarian carcinomas are usually positive for cytokeratin 7, often positive for ER and typically negative for cytokeratin 20 . The epithelial membrane antigen expression pattern is typical for serous papillary carcinoma, with expression on the outside of the papillary clusters and around the central spaces [6]. GCDFP-15 expression is rarely observed in ovarian carcinoma, whereas staining for cancer antigen CA 125 is present in $\sim 60 \%$ and $90 \%$ of ovarian and serous papillary carcinomas, respectively [6]. CA125 expression is also normally observed in endocervical, endometrial, pancreatic and biliary carcinomas, but is less typically present in breast cancer. Another immunohistochemical marker is mesothelin, which is expressed in $>90 \%$ of serous papillary carcinomas of the ovary, but is weakly expressed in $3-14 \%$ of breast cancer cases. Lung, colorectal and gastric adenocarcinomas present with intermediate levels of staining [5].

The incidence of extramammary metastases is increasing due to the improvements in treatment leading to longer survival. Unusual sites of metastases are more and more reported. In addition, the frequent use of modern imaging techniques like PET-CT leads to the detection of subclinical lesions.

According to several studies, after the detection of metastatic breast disease secondary to an ovarian primary tumor, survival times ranged from 13 days to 3.5 years, with most patients dying within 1 year [8].

The prognosis of MOCB has been described as poor, which is attributed to the fact that MOCB represents a late stage of ovarian cancer progression. Palliative surgery with mastectomy may be considered for those unresponsive to chemotherapy [3].

\section{Conclusion}

The possibility of breast metastasis should be considered in patients with a history of advanced malignant disease in case of a lump breast. Definite diagnosis should be made from not only laboratory and imaging studies but also clinical presentation. When the clinical finding and morphology are obscure, immunohistochemistry study plays a major role in the differential diagnosis. A panel of WT1, PAX8, and GCDFP-15 immunoperoxidase seems to be helpful in the diagnosis.

The combination of a clinical acumen, radiology, histopathology and immunohistochemistry is imperative in the management of such a case to avoid misdiagnosis.

\section{References}

1. Achariyapota V, Chuangsuwanich $T$, Benjapibal $M$ 
(2016) Case Report Inflammatory Breast Cancer from Metastatic Ovarian Cancer. Case Reports in Obstetrics and Gynecology 2016: 1-4.

2. Galili Y, Lytle M, Bartolomei J, Amandeep K, Allen N, et al. (2019) Case Report Clear-Cell Carcinoma of the Ovary with Bilateral Breast Metastases. Case Rep Oncol Med 2019: 1-5.

3. https://gco.iarc.fr/today/data/factsheets/populations/900world-fact-sheets.pdf

4. Fauzi B, Attrache E, Highsmith C, Alan BG, Kapenhas E (2017) A Rarity in Breast Disease: Metastatic Ovarian Carcinoma to the Breast Mimicking Inflammatory Breast Cancer. J Univer Surg 5: 3.

5. Antuono L, Angela F, Luca N, Giovanni M, Enrico C (2018) Breast metastasis from ovarian cancer: A case report. Radiol Case Rep 13: 1166-1169.

6. Tempfer CB, Fizazi NEL, Ergonenc H, Solass W (2016) Metastasis of ovarian cancer to the breast : A report of two cases and a review of the literature. Oncol Lett 11: 40084012.
7. Klein RL, Brown AR, Gomez-Castro CM, Chambers SK, Cragun JM, et al. (2010) Ovarian cancer metastatic to the breast presenting as inflammatory breast cancer: A case report and literature review. Journal of Cancer Ovarian Cancer 1: 27-31.

8. Moore DH, Wilson DK, Hurteau JA, Look KY, Stehman FB, et al. (1998) Gynecologic cancers metastatic to the breast. J Am Coll Surg 187: 178-181.

9. DeLair DF, Corben AD, Catalano JP, Vallejo CE, Brogi E, et al. (2013) Non mammary metastases to the breast and axilla: A study of 85 cases. Mod Pathol 26: 343-349.

10. Karam AK, Stempel M, Barakat RR, Morrow M, Gemignani ML (2009) Patients with a history of epithelial ovarian cancer presenting with a breast and/or axillary mass. Gynecol Oncol 112: 490-495.

11. Mori R, Futamura M, Morimitsu K, Saigo C, Miyazaki T, et al. (2017) The diagnosis of a metastatic breast tumor from ovarian cancer by the succession of a p53 mutation: A case report. World J Surg Onc 15: 117. 